\title{
Combining Microarray Technology and Molecular Epidemiology to Identify Genes Associated with Invasive Group B Streptococcus
}

\author{
Lixin Zhang, ${ }^{1}$ Usha Reddi, ${ }^{2}$ Usha Srinivasan, ${ }^{1}$ Sheng Li, ${ }^{1}$ Stephanie M. Borchardt, ${ }^{1,}{ }^{3}$ Parvathy Pillai, ${ }^{1}$ \\ Puja Mehta, ${ }^{1}$ Anne N. Styka, ${ }^{1}$ Joan DeBusscher, ${ }^{1}$ Carl F. Marrs, ${ }^{1}$ and Betsy Foxman ${ }^{1}$ \\ ${ }^{1}$ Department of Epidemiology, University of Michigan School of Public Health, Ann Arbor, MI 48109, USA \\ ${ }^{2}$ Program in Bioinformatics, Eastern Michigan University, Ypsilanti, MI 48197, USA \\ ${ }^{3}$ Fargo VA Medical Center, Fargo, ND 58102, USA
}

Correspondence should be addressed to Lixin Zhang, lxzhang@umich.edu

Received 29 August 2007; Accepted 29 November 2007

Recommended by Joshua P. Metlay

\begin{abstract}
Many bacterial species function as both commensals and pathogens; we used this dual nature to develop a high-throughput molecular epidemiological approach to identifying bacterial virulence genes. We applied our approach to Group B Streptococcus (GBS). Three representative commensal and one invasive GBS isolates were selected as tester strains from a population-based collection. We used microarray-based comparative genomic hybridization to identify open reading frames (ORFs) present in two sequenced invasive strains, but absent or divergent in tester strains. We screened 23 variable ORFs against 949 GBS isolates using a GBS Library on a Slide (LOS) microarray platform. Four ORFs occurred more frequently in invasive than commensal isolates, and one appeared more frequently in commensal isolates. Comparative hybridization using an oligonucleotide microarray, combined with epidemiologic screening using the LOS microarray platform, enabled rapid identification of bacterial genes potentially associated with pathogenicity.
\end{abstract}

Copyright ( 2008 Lixin Zhang et al. This is an open access article distributed under the Creative Commons Attribution License, which permits unrestricted use, distribution, and reproduction in any medium, provided the original work is properly cited.

\section{INTRODUCTION}

Group B Streptococcus (GBS), or Streptococcus agalactiae, a common bowel inhabitant, also frequently colonizes the vagina, urethra, and pharynx asymptomatically. However, GBS can cause a variety of invasive diseases that occur primarily in neonates, young infants, elderly persons, and pregnant women [1-3]. Host factors are clearly important as GBS disease occurs primarily in vulnerable populations. Nonetheless, bacterial virulence factors must also play a role: propensity to cause disease varies by serotype [4], and genetic sequence types that cross serotype, which have a high potential to cause invasive disease, have been identified [5].

Among the nine known GBS capsular serotypes, serotypes Ia, III, and V cause the majority of GBS disease in the United States [1, 6-8]. Studies of the population structure and the molecular epidemiology of GBS isolates suggest that GBS populations are clonal, but that some strains may be more virulent than others [9-13]. By pulsed-field gel electrophoresis (PFGE), disease-causing isolates have limited heterogeneity within a serotype (reviewed in Schuchat, 1998 [2]) while colonizing isolates are quite heterogeneous [14] within a particular serotype, suggesting that invasive isolates have distinctive features which enhance pathogenesis. Differences in virulence are likely related to the presence or absence of virulence genes [15]. Although advances have been made in the understanding of classic GBS traits, such as capsular polysaccharide, $\beta$-hemolysin, C5a peptidase, and immunogenic surface proteins [16-19], our understanding of the pathogenesis of GBS infections is limited: little is known about which bacterial genetic factors contribute to virulence or transmission of pathogenic strains.

The availability of complete and draft genome sequences of several GBS strains presents an opportunity to gain insight into the molecular basis of GBS virulence. Analysis of these genome sequences confirmed a high level of genetic heterogeneity among GBS strains, even of the same serotype [20]. The GBS genome contains a large number of genetic islands 
that often vary among strains and are likely to be the regions where virulence genes reside [21-23]. With thousands of genes identified in GBS genomes, the current challenge is to determine which are important in GBS pathogenesis and transmission. In a previous study of $E$. coli, we presented an approach of bacterial gene identification and evaluation that relied on epidemiologic information for selecting isolates for genomic subtraction and screening of epidemiologically defined collections for evaluation of the significance of genes identified through genomic subtraction [24]. In this report, we applied the same principle in a three-step strategy to the study of GBS. Further, we employed novel microarray platforms that enabled us to systematically identify candidate genes and evaluate their importance on a large scale.

Genome comparison between pathogenic and nonpathogenic strains within a species is a powerful strategy for identifying candidate genes important for virulence $[25,26]$. Since none of the currently sequenced GBS genomes are from commensal isolates, we first selected a few representative colonizing GBS strains from a population-based sample for comparisons with sequenced pathogenic serotype III strain (NEM316) and serotype V strain (2306VR), two serotypes representing the most frequently encountered disease-causing isolates. We then identified sequence differences and their associated variable genes between selected colonizing and sequenced invasive GBS strains using comparative genomic hybridization with fine-tiling oligonucleotide microarrays. Lastly, a selected set of variable genes was screened against a large panel of colonizing and invasive strains using Library on a Slide microarray to evaluate their association with disease. Our main objective of this report is to use GBS as an example to demonstrate and evaluate this study approach.

\section{MATERIALS AND METHODS}

\subsection{Bacterial strains and culture conditions}

Disease-causing and commensal GBS isolates were selected from various collections obtained from previous epidemiologic studies. Collections included isolates from healthy male and nonpregnant female college students enrolled at the University of Michigan [27-30], isolates from symptomatic and asymptomatic pregnant women seen at a University of Michigan Medical Center clinic [31], and isolates collected from patients through the Wisconsin Invasive Bacterial Laboratory Surveillance System between 1998 and 2002 [32]. Additional isolates from newborns in Texas with early and late onset disease [8] as well as isolates from pregnant women with and without GBS disease were obtained from Dr. Carol J. Baker (Baylor College of Medicine, Houston, Texas, USA). These strains were broadly grouped into two categories: invasive isolates from patients with invasive diseases $(n=386)$ and colonizing isolates from subjects without any symptomatic diseases $(n=563)$. GBS strain NEM316 [21], 2603VR [23], and A909 [33] were used as reference strains. GBS isolates were cultured overnight in Todd-Hewitt broth (Oxoid) for DNA isolation.

\subsection{PFGE and capsular typing}

PFGE was performed as described previously [30]. Briefly, GBS DNA was digested with SmaI and electrophoresed for 18 hours (initial switch time 4 seconds; final switch time $16 \mathrm{sec}$ onds) with the CHEF III apparatus (Bio-Rad, Hercules, CA). Gels were strained for $4 \mathrm{~h}$ with Vistra Green (Amersham Biosciences, Piscataway, NJ, USA) at $4 \mathrm{C}$, and visualized with a Storm PhosphorImager (Amersham Biosciences, Piscataway, NJ, USA). PFGE patterns were analyzed using BioNumeric software (Applied Maths, Kortrijk, Belgium). A dendrogram was constructed using the unweighted pair group method with arithmetic means, Dice coefficient, optimization setting of $1.0 \%$, and a position tolerance of $1.0 \%$. GBS isolates were classified into capsular types Ia, Ib, and II-VIII using DNA dot blot hybridization, as previously described [34].

\subsection{GBS oligonucleotide microarray construction}

Two fine-tiling oligonucleotide microarrays were designed using the published DNA sequences for serotype III strain (NEM 316, GenBank accession no. AL732656) and serotype V strain (2603VR, GenBank accession no. AE009948). The NEM 316 array consisted of a total of 368,576 32-mer probes (184,288 pairs) tiling its $2.21 \mathrm{Mb}$ genome every 12 bases for both strands. The 2603VR array consisted of a total 360,040 32 -mer probes (180,020 pairs) tiling its $2.16 \mathrm{Mb}$ genome every 12 bases for both strands. Arrays were designed and constructed through custom Array CGH service from NimbleGen (Madison, Wiss, USA) using its maskless array synthesis (MAS) technology. A denser tiling array with shorter oligonucleotides was usually used in NimbleGen's two-step comparative genome sequencing (CGS) [35].

\subsection{Comparative genome hybridization and data acquisition}

Comparative genomic hybridization and signal processing were performed by NimbleGen custom service (NimbleGen Systems Inc., Madison, Wiss, USA). Briefly, GBS DNA from four tester strains and two reference strains were broken down into separate pools of low molecular weight fragments, labeled independently with cyanine fluorescent dye and each was hybridized to one NEM316 and to one 2603VR wholegenome tiling array. Similar to the Affymetrix chips, the short oligo GBS arrays were produced by in-slide de novo syntheses. Like Affymetrix chip hybridization, one slide was used for hybridization per labeled sample. A total of 12 microarray hybridizations were performed. Genomic hybridization of NEM316 or 2603VR to its own array served as a signal reference for comparisons with tester strain hybridizations using the same array. Genome hybridization of one sequenced genome against the other sequenced genome array was used for validation purposes. The signal intensity ratios of tester DNA to each reference DNA were compared to identify probe sequences absent or different from the tester genome. The ratio was generated by normalizing the signal intensity (setting the median ratio to 1 and the standard deviation to 0.45 ), dividing the reference by the tester for each strand, and then 
averaging the two strands. Signal ratios were plotted as a function of their genomic positions and visualized using SignalMap software from NimbleGen. A custom algorithm was used to mark the potential variable probe sequence (absent or different in tester genome) based on comparison to a local threshold (a $1800 \mathrm{bp}$ window). This analysis was also performed by NimbleGen (see Supplementary Material available at doi:10.1155/2007/14762 for the analytical algorithm).

\subsection{Additional bioinformatic and data analysis methods}

Genome-scale sequence comparisons between genomes of NEM316 and 2603VR were performed using GenomeComp [36]. All strain-specific genetic islands greater than $10 \mathrm{bp}$ were identified using run parameters set to $0.01,-3$, and 1 for expectation value (e), penalty for a mismatch, and reward for a match, respectively. To identify matches of probe sequences from one fine-tiling oligoarray in the other sequenced genome, a custom Bioperl program was used for local batch blast analyses. The stand alone BLAST program [37] for Unix operation system was used and the percent identity of the best hit for each probe within the query genome was determined. All other data analyses were performed using SAS v9.0 (SAS Institute, Inc Cary, NC, USA) and S plus v6.1 (Insightful Corporation, Seattle, Wash, USA).

\subsection{GBS library on a slide microarray construction and hybridization}

We recently developed a new application of microarray technology, called Library on a Slide (LOS), for bacterial comparative genomics studies [38]. LOS technology combines dot blot hybridization with the technology of microarrays resulting in glass slides with thousands of bacterial genomes arrayed. Thus libraries of entire genomes rather than the sequence of a single genome or set of genes are printed on the slides. Slides are used to screen large numbers of strains for the presence of specific genetic elements of interest. A GBS LOS microarray was created with genomic DNA from 949 GBS isolates sampled from a variety of GBS collections and various control strains. Genomic DNA was isolated using a high-throughput sonication-based method described previously [39]. DNA from GBS strains along with controls were arrayed in duplicate on Vivid Gene Array slides (Pall Life Sciences, Mich, USA) using a VersArray ChipWriter compact arrayer (Bio-Rad, Calif, USA). Selected GBS ORFs and four house keeping genes, (alcohol dehydrogenase $(a d h P)$, phenylalanyl tRNA synthetase (pheS), glutamine synthetase $(g \ln A)$, and glucose kinase $(g l c K))$, were PCR-amplified from either strain NEM316 or 2603VR. Purified PCR products were fluorescein-labeled using BioPrime DNA labeling kit (Invitrogen, Calif, USA). Each probe was hybridized with a different slide overnight at $68^{\circ} \mathrm{C}$ in PerfectHyb Plus hybridization buffer (Sigma, Mo, USA). After washing, fluorescein-labeled probes were detected using antiflourescein alkaline phosphatase (Roche, Switzerland) and alkaline phosphatase kit (TeleChem, Calif, USA). The intensity of each spot was normalized to the intensity of the quan- tification probe (a mixture of four housekeeping genes) to account for differences in DNA concentrations at different spots, and compared to the intensity of the positive control (sequence strain known to contain the gene probe) to determine presence/absence of the gene fragment in different bacterial strains using previously established methods $[38,40]$.

\section{RESULTS AND DISCUSSION}

\subsection{Selection of tester strains for comparative genomic subtraction.}

Comparing pathogenic and nonpathogenic strains within a species can provide critical insights into bacterial pathogenesis. However, all sequenced GBS strains are from invasive diseases. We used molecular epidemiological comparisons to select representative commensal colonizing GBS isolates for the comparative genomic hybridization with the highest potential to identify potential pathogenesis-related genes in sequenced invasive genomes (strains NEM316 and 2603VR). We characterized the diversity of 882 colonizing isolates from a population-based longitudinal study of healthy male and nonpregnant female college students [28] using PFGE and serotyping. Clustering analysis by dendrogram was performed on these isolates along with a sample of 35 invasive isolates and sequenced pathogenic strains NEM316 and 2603VR. Based on this analysis, we selected three commensal isolates that are genetically distant from the two sequenced genomes but representative of isolates from relatively large strain clusters that are predominantly of commensal origin. Isolate $657-461$ is a serotype $V$ strain representing the largest clonal group within our commensal collection. Isolate G617061 (serotype III) and G293-061 (serotype II) are from two additional clusters dominated by colonizing strains. In addition to these three commensal isolates, one invasive isolate, $\mathrm{H}-19$, was chosen for the comparative genomic subtraction, because it represents the most common clonal type of the serogroup Ia strain in our analysis. Since serotype classification does not necessarily reflect genetic distance among strains [20], we did not select tester strains based solely on the differences in serotype. The small number of colonizing stains chosen here could not and was not intended to capture the diversity of commensal isolates. It was our first attempt at performing genome-wide comparisons between colonizing strains and sequenced genomes, so we could pick gene candidates from a list of several thousands for association study using LOS microarrays where large collections of population-based GBS isolates can be screened.

\subsection{Oligonucleotide array validation}

We used shared probe sequences within the two genome arrays to assess the reproducibility of the comparative genomic hybridization and used hybridization of one sequenced genome against the array of the other sequenced genome to evaluate the accuracy of the array in assessing sequence variation.

Between 184,288 probe pairs on the NEM316 array and 180,020 probe pairs on the $2603 \mathrm{VR}$ array, a total of 16,364 
TABLE 1: Sensitivity (probability open reading frame is detected, given it is truly present) and specificity assessments (probability open reading frame is not detected, given it is not present) of different cutoff values in classifying variable open reading frames using fine-tiling oligonucleotide genome arrays created from the genomic sequence of group B Streptococcus strains NEM316 and 2603 VR.

\begin{tabular}{lccc}
\hline Reference genome & Percentage cutoff point & Sensitivity & Specificity \\
\hline \multirow{2}{*}{ NEM316 } & $20 \%$ & 0.98 & 0.91 \\
& $15 \%$ & 0.97 & 0.94 \\
& $10 \%$ & 0.96 & 0.96 \\
2603 VR & $20 \%$ & 0.97 & 0.91 \\
& $15 \%$ & 0.95 & 0.92 \\
\hline
\end{tabular}

TABLE 2: Number (percentage) of variable probe sequences among four tester group B Streptococcus genomes using sequenced strains as a reference revealed by comparative genomic hybridization

\begin{tabular}{lcccc}
\hline $\begin{array}{l}\text { Reference/sequenced } \\
\text { genomes of invasive } \\
\text { isolates }\end{array}$ & $\begin{array}{c}\text { H1-19 (Ia) } \\
\text { (invasive) }\end{array}$ & $\begin{array}{c}\text { G293-061 } \\
(\text { II) } \\
\text { (commensal) }\end{array}$ & $\begin{array}{c}\text { G617-061 } \\
(\text { III) } \\
\text { (commensal) }\end{array}$ & $\begin{array}{c}\text { G654-461 } \\
(\text { V) }\end{array}$ \\
\hline 2603VR & $327(15.4 \%)$ & $320(15.1 \%)$ & $72(3.4 \%)$ & $278(13.1 \%)$ \\
NEM316 & $277(13.0 \%)$ & $305(14.3 \%)$ & $305(14.3 \%)$ & $271(12.7 \%)$ \\
\hline
\end{tabular}

identical probe pairs (32/32 match) were identified. Hybridization results for this probe subset from two arrays for each tester genome were treated as replicas for accessing the reproducibility of $\mathrm{CGH}$. Hybridization for each probe was classified as identical or variable in the tester genome when compared to the reference genome. The percent concordance for tester genomes G293-061, H1-19, G617-061, and G654461 were $98.75 \%, 99.50 \%, 99.88 \%$, and $98.62 \%$, respectively. The reproducibility was very high even when the raw signal ratios were examined. The correlation coefficients were greater than $90 \%$ for duplicates.

To evaluate the accuracy of the array in assessing sequence variation, we compared results from in silicoanalysis with the classification results from an actual array hybridization swap between NEM316 and 2603VR genomes. Almost all perfectly matched probe sequences were correctly identified as identical by hybridization. Only 4 out of 133,520 and 5 out of 133,570 probe sequences were falsely identified as different with NEM316 and 2603VR arrays, respectively. However, 28,673 out of 50,768 (56\%) and 25,435 out of $46,450(55 \%)$ mismatched probe sequences were falsely identified as identical with NEM316 and 2603VR arrays, respectively. At the probe level, hybridization has a high sensitivity but low specificity for detecting conserved probe sequences. Nonetheless, the high-density nature of the tiling array still provides overall sequence variation information at genome and ORF levels. We visually displayed the CGH results by plotting hybridization signal ratios of the probes along their genomic positions and compared them with an in silico comparison of NEM316 and 2603VR. The majority of variable probes (i.e., probes with high reference versus tester signal ratios) are clustered primarily around strain-specific genetic islands identified by the in silico analysis. To convert probe-level variation to ORF sequence variation, we calculated the percentage of variable probes for each ORF (number of variable probes identified within an ORF divided by the total number of probes tiling the ORF). Using different percentage cutoff values in classifying variable ORFs (divergent or absent), the CGH-based data was compared with in silico analysis (Table 1). For the NEM 316 array, a 15\% cutoff value gave a $2.9 \%$ false-negative rate (i.e., ORFs known to be present but classified by hybridization as absent or very divergent) and the best overall sensitivity and specificity (97\% and $94 \%$, resp.). For the $2603 \mathrm{VR}$ array, the $20 \%$ cutoff point gave the best overall sensitivity and specificity (97\% and $91 \%$, resp.). Thus, these two cutoff points were chosen to classify variable ORFs for the remaining $\mathrm{CGH}$ analyses.

Comparative genomic hybridization with fine-tiling 32mer oligonucleotide microarrays did not identify all probe sequence variations in the two reference genomes but reliably identified variable ORFs using combined hybridization results of all probes within each ORF.

\subsection{Distribution and mapping of variable probe sequences}

CGH using NEM316 and 2603VR genome arrays revealed that $3.4-15.4 \%$ of probe sequences were absent or divergent in the four tester strains (Table 2). This range of diversity is similar to the range of sequence differences (5\% to $15 \%)$ recently observed in pairwise comparison of all eight available complete or draft GBS genome sequences [20]. The tiling arrays allowed a high-resolution view of genome variation among comparison strains. Figure 1 displays comparative hybridization results of tester strains against 2603VR by plotting the reference to tester signal ratios along their genomic positions. Although the four tester strains represent four different serotypes (Ia, II, III and V), the majority of absent or divergent probe sequences in these strains are mapped to the same set of regions on the NEM316 genome, a serotype III strain. To a lesser extent, the same set of regions on the 2603VR genome covers the majority of the probes that are absent or divergent across three of the four tester genomes. The strain G617-061, a serotype III strain, is very similar to 


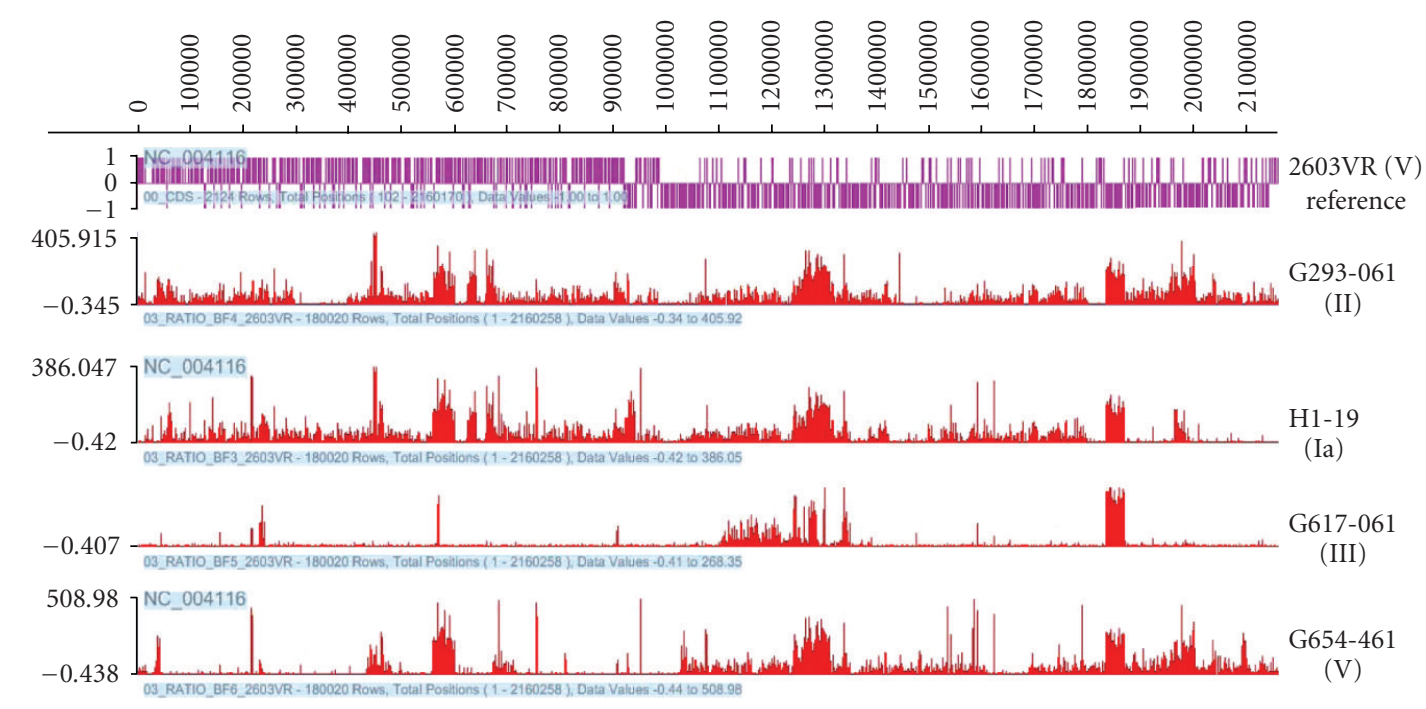

FIGURE 1: Locations of variable probes sequences identified within genomes of group B Streptococcus strains 2603VR in comparative genomic hybridization using each of the four tester strains of group B Streptococcus.

the serotype V reference strain 2603VR genome, with only $3.4 \%$ probes identified as different compared to $>13 \%$ of the other tester genomes. Comparison of all eight available complete or draft GBS genome sequences also demonstrated that serotype classification does not reflect the genetic diversity of the GBS [20]. One possible explanation for closely related strains exhibiting different capsules is genetic exchange of genes determining the capsular type by horizontal gene transfer.

\subsection{NEM316 and 2603VR ORFs absent/divergent in tester genomes}

Hybridization results from all the probes within each ORF were used to determine the presence or absence/divergence in the tester genome using the criteria established through analyzing control experiments (described above). Among the 2134 ORFs within the NEM316 genome, $484(22.7 \%)$ were identified as variable ORFs because they were classified as absent/divergent in at least one tester genome. 269 (56\%) of them were absent/divergent in four tester genomes, and 96, 84, and 35 were classified as absent/divergent in 1,2 , and 3 genomes, respectively. Of 2124 ORFs within the 2603VR genome, $530(25 \%)$ were identified as variable ORFs. Among them, 81, 121, 162, and 166 were classified as absent/divergent in 4, 3, 2, and 1 tester genomes, respectively. Pairwise genome alignment of the two reference genomes identified strain-specific regions with a total length of $288 \mathrm{~kb}$ and $239 \mathrm{~kb}$ in NEM316 and 2603VR, respectively. Greater than $95 \%$ of the ORFs residing within these strain-specific regions were identified as variable ORFs in our CGH with four tester genomes, representing 64\% (309/484) of NEM316 variable ORFs and 52\% (275/530) of the $2603 \mathrm{VR}$ variable ORFs. About $80 \%$ of variable ORFs identified by CGH are located within fourteen putative pathogenicity islands previously identified in NEM316 [22].
To investigate which functional groups these variable ORFs belong to, we classified ORFs into clusters of orthologous genes (COGs) [41]. Figure 2 shows the number of variable ORFs in each COG category. Approximately half of the variable ORFs have not been classified into COGs and are of unknown function. The most common classifiable variable ORFs belong to the COG category of DNA replication, recombination, and repair. This is likely attributable to the presence of integrated phage or plasmids in the reference genomes. A large number of variable ORFs are predicted to be involved in transport, regulation, intermediate metabolism, and cell wall metabolism. These genes may be important in maintaining the pathogenic life style and transmission of the invasive GBS strains. Genes within these categories have also been identified through an in vivo study in which signature-tagged mutagenesis and a neonatal rat model were used to identify novel GBS genes implicated in virulence [42]. Relatively few variable ORFs were found to be involved in coenzyme transport and metabolism and lipid transport and metabolism.

\subsection{ORFs absent/divergent in at least two tester genomes of commensal origin}

ORFs consistently absent/divergent in commensal tester strains compared to invasive strains are likely virulence gene candidates. Six ORFs were absent/divergent in all three commensal tester strains, and conserved in the two invasive reference genomes and the invasive tester strain. We identified an additional 29 ORFs from the reference genomes that were absent/divergent in at least two out of the three commensal strains (Table 3). Fifteen of these 35 ORFs are predicted hypothetical to be proteins of unknown function. Several ORFs are predicted to be proteins involved in transport, metabolism, and other metabolic functions. Also included are two putative lipoproteins and two surface proteins. Gene 


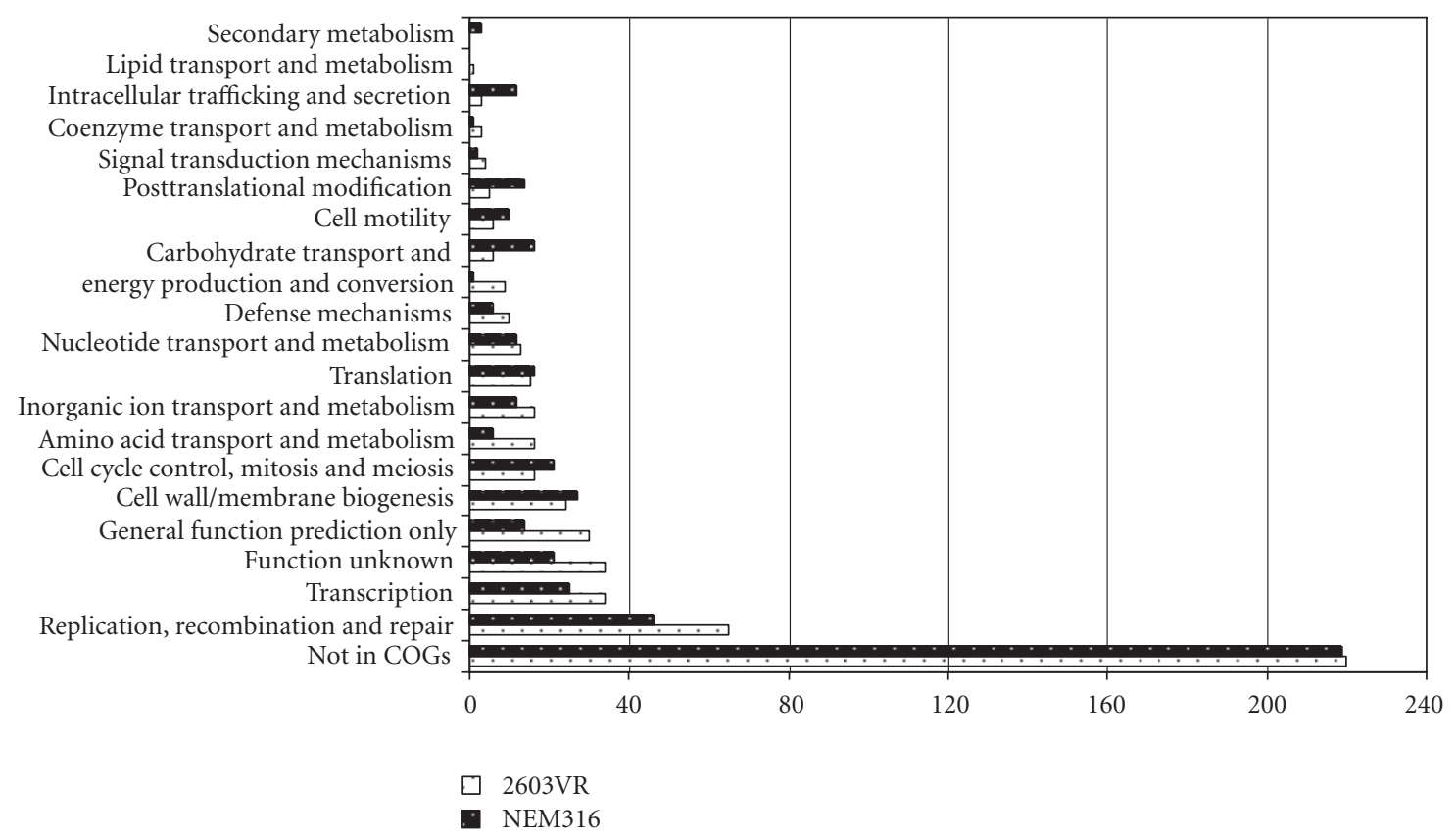

FIGURE 2: Number of variable open reading frames in group B Streptococcus strains NEM316 and 2603VR classified in each of the clusters of orthologous genes (COG) category plus those not classified in COG database.

gbs0850 is predicted to encode a fibrinogen binding protein and is identical to a previously identified $f b s B$ gene $[43,44]$. The best match for gbs0850 in $2603 \mathrm{VR}$ is ORF sag0832, which encodes a different variant of the fibrinogen binding protein. ORFs gbs2015 and gbs2016 are two adjacent genes with highly similar DNA sequences predicted to encode glycosyl transferases. These two genes are also found together in the 2603 VR genome as sag2060 and sag2061.

While we identified a large number of variable ORFs, few were missing in all three or even two commensal tester strains. Because multiple genetic factors are involved in GBS virulence and multiple pathogenesis pathways involving different sets of virulence genes likely exist, we might expect different sets of virulence genes to be identified when different pairs of invasive and colonizing isolates are compared.

\subsection{GBS library on a slide hybridization and differentially distributed variable ORFs}

All variable ORFs are potential virulence gene candidates. Using a novel GBS LOS microarray platform with a large collection of GBS isolates, the importance of these variable ORFs can be efficiently evaluated. We present our initial evaluation of 23 of the 35 ORFs identified above using GBS LOS. We were not able to synthesize good probes with strong and specific signals in our initial attempts for the other 12 variable ORFs because of their small sizes or poor PCR amplifications. We therefore left them out of this initial LOS screening. The GBS LOS microarray contained genomic DNA from 949 GBS isolates printed in duplicate. Among them, 386 were isolates from patients with invasive diseases and 563 were commensal colonizing isolates. In addition, the LOS array contained DNA from various control strains. Table 3 lists the prevalence of 23 ORF in the overall GBS collection and their prevalence ratios in invasive strains compared to colonizing strains. sag2060, sag2061, sag0832, and gbs0474 appeared more frequently in invasive isolates than in colonizing commensal strains. By contrast, sag0814 was more frequently found among colonizing isolates than invasive isolates.

ORFs sag2060 and sag2061 are two putative glycosyl transferase genes. Glycosylation plays an important role in many biological processes in eukaryotes, and there is increasing evidence for a role of glycosylation in bacteria. Many surface expressed bacterial structures such as LPS, LOS, capsule, flagella, and pili in pathogenic bacteria are glycosylated [45-47]. Glycosylation can also be used by bacteria to inactivate antibiotics $[48,49]$. Interestingly, the glycosyl transferase gene lic $2 B$ in Haemophilus influenzae was found more frequently among isolates causing otitis media than in throat isolates from children [50]. Future mechanistic studies should shed light on the roles of these GBS glycosyl transferases in pathogenesis.

The sag0832 is predicted to encode a fibrinogen binding protein. This gene has been suggested to be an important virulence gene for invasive GBS disease. In a murine model of sepsis, the wild-type strain was more virulent than the isogenic strain with this gene inactivated [44]. sag0832 was also shown to promote GBS invasion into epithelial cells in vitro [43]. In addition to the three ORFs encoding known proteins, two ORFs (sag0814 and gbs0474) encoding hypothetical proteins were differentially distributed between invasive and colonizing strains. Given the large numbers of ORFs of no known functions that exist in the sequenced genomes, this is not surprising. Some of these ORFs probably involve complicated traits that are difficult to observe in laboratory conditions and will be more readily identified using association 
TABLE 3: Open reading frames (ORF) present in invasive strains but absent at least in two out of three commensal tester group B Streptococcus strains by comparative genomic hybridization and their presence among 949 GBS isolates and their prevalence ratio between invasive $(n=$ 386) and colonizing $(n=563)$ isolates.

\begin{tabular}{|c|c|c|c|}
\hline ORF & Probe-positive strains (\%) & Prevalence ratio $(95 \% \mathrm{CI})^{(\mathrm{a})}$ & Predicted protein \\
\hline sag0004 & $524(55 \%)$ & $1.1(0.96-1.22)$ & hypothetical protein \\
\hline sag0005 & $706(74 \%)$ & $1.0(0.93-1.09)$ & hypothetical protein \\
\hline sag0027 & $941(99 \%)$ & $1.0(0.98-1.00)$ & $\begin{array}{l}\text { phosphoribosylaminoimidazole } \\
\text { synthetase }\end{array}$ \\
\hline sag0175 & $692(73 \%)$ & $1.0(0.93-1.09)$ & hypothetical protein \\
\hline sag0206 & $590(62 \%)$ & $0.9(0.82-1.01)$ & lipoprotein, putative \\
\hline sag0253 & (b) & (b) & acetyltransferase, GNAT family \\
\hline sag0414 & $927(98 \%)$ & $1.0(0.97-1.01)$ & phosphorylase, Pnp/Udp family, putative \\
\hline sag0426 & - & - & cupin family protein \\
\hline sag0427 & $517(54 \%)$ & $1.0(0.93-1.17)$ & transcriptional regulator, MerR family \\
\hline sag0700 & $925(97 \%)$ & $1.0(0.97-1.02)$ & $\begin{array}{l}\text { 2-dehydro-3-deoxyphosphogluconate } \\
\text { aldolase/4-hydroxy-2-oxoglutarate } \\
\text { aldolase }\end{array}$ \\
\hline sag0814 & $117(12 \%)$ & $0.6(0.39-0.83)$ & hypothetical protein \\
\hline sag0815 & $364(38 \%)$ & $0.9(0.76-1.06)$ & $\begin{array}{l}\text { transcriptional regulator, Cro/CI } \\
\text { family-related protein }\end{array}$ \\
\hline sag0832 & $371(39 \%)$ & $1.5(1.29-1.77)$ & fibrinogen binding protein \\
\hline sag1130 & $367(39 \%)$ & $1.1(0.90-1.25)$ & hypothetical protein \\
\hline sag1140 & (b) & (b) & hypothetical protein \\
\hline sag1207 & (b) & (b) & hypothetical protein \\
\hline sag1781 & (b) & (b) & primase-related protein \\
\hline sag1968 & $87(9 \%)$ & $1.1(0.71-1.61)$ & hypothetical protein \\
\hline sag1969 & $907(96 \%)$ & $1.0(0.97-1.03)$ & ribosomal protein L11 methyltransferase \\
\hline sag1974 & (b) & (b) & MutT/nudix family protein \\
\hline sag1975 & (b) & (b) & hypothetical protein \\
\hline sag1976 & $290(31 \%)$ & $0.9(0.73-1.08)$ & hypothetical protein \\
\hline sag1994 & $289(30 \%)$ & $1.0(0.82-1.22)$ & hypothetical protein \\
\hline sag1999 & (b) & (b) & hypothetical protein \\
\hline sag2021 & $395(42 \%)$ & $1.2(1.00-1.36)$ & cell wall surface anchor family protein \\
\hline sag2026 & $224(24 \%)$ & $1.1(0.88-1.40)$ & membrane protein, putative \\
\hline sag2027 & (b) & (b) & $\mathrm{ABC}$ transporter, ATP-binding protein \\
\hline sag2028 & (b) & (b) & hypothetical protein \\
\hline sag2045 & $364(38 \%)$ & $1.1(0.90-1.25)$ & $\begin{array}{l}\text { DNA topology modulation protein FlaR, } \\
\text { putative }\end{array}$ \\
\hline sag2057 & (b) & (b) & leucyl-tRNA synthetase \\
\hline sag2060 & $427(45 \%)$ & $1.3(1.13-1.50)$ & glycosyl transferase, family 8 \\
\hline sag2061 & $437(46 \%)$ & $1.2(1.07-1.41)$ & glycosyl transferase, family 8 \\
\hline sag2088 & (b) & (b) & hypothetical protein \\
\hline sag2147 & $687(72 \%)$ & $1.0(0.9-1.06)$ & lipoprotein, putative \\
\hline gbs0474(c) & $270(28 \%)$ & $1.5(1.21-1.80)$ & hypothetical protein \\
\hline
\end{tabular}

\footnotetext{
(a) Prevalence with confidence interval not overlapping 1 are considered statistical significant.

(b) These ORFs were not screened using LOS mostly because of small sizes.

(c) Exact corresponding gene in strain 2603VR was not found by blast search while it was classified as present in 2603 VR by CGH.
}

studies. Interestingly, sag0814 is found more frequently in commensal strains than in invasive strains. It is possible that lack of this gene enhances virulence. During the process of commensal-to-pathogen evolution, bacteria not only acquire virulence genes but also shed genes via deletions [51]. Dele- tions of genes that facilitate a commensal lifestyle could provide an additional evolutionary pathway towards virulence. For example, deletion of lysine decarboxylase gene greatly enhanced the enterotoxin activity in Shigella in its evolution [51]. 
While five out of 23 variable ORFs were differentially distributed between invasive and commensal isolates, the associations of these ORFs with invasive isolates were neither exclusive nor strong. Such outcomes were not unexpected for several reasons. First, we anticipate some degree of random misclassification to decrease the observed associations because invasive strains can be also commensal, and noninvasive strains can become opportunistic pathogens. Second, similar to the existence of several distinct pathotypes within many bacterial pathogens, there may be many different pathotypes within GBS. One virulence gene may be strongly associated with strains within one specific GBS pathotype but the association is less pronounced when all invasive isolates are included in the analysis. Third, GBS pathogenesis is determined by not one but many virulence genes and any one gene may only contribute. We are in the process of screening these and more variable ORFs on an additional 2000 isolates in order to perform a more definitive analysis. In addition, incorporating the population structure of GBS could potentially enhance our analysis and help with interpretation.

Screening 23 ORFs against 949 isolates also revealed the striking genome content diversity of GBS. We assigned each isolate to a genotype based on the presence or absence of all 23 probed examined. A total of 503 genotypes were observed among 949 isolates. Using this classification, strains with the same PFGE patterns have different gene composition. Profiling GBS with a limited number of gene probes could therefore provide a highly discriminative typing method.

\section{CONCLUSIONS}

As increasing numbers of bacterial genomes are sequenced, postgenome research will focus on identifying virulencerelated genes and the function of these genes. We used a three-step molecular epidemiological approach employing two novel microarray platforms, fine-tiling oligonucleotide microarrays, and Library on a Slide to identify bacterial virulence genes potentially contributing to GBS disease. Among hundreds of variable ORFs identified by $\mathrm{CGH}$, 35 were absent/divergent in two of out three commensal test strains but present in two invasive reference genomes and a tester invasive strain. We screened 23 of these ORFs against 949 GBS isolates, and found 5 ORFs that were differentially distributed between invasive and commensal isolates. We demonstrated that this approach can rapidly identify and evaluate bacterial genes potentially associated with pathogenicity.

In our approach, we adopted microarray-based CGH instead of the traditional genomic subtraction method to identify genetic differences between paired commensal and invasive GBS strains. The traditional genomic subtraction approach can sample only a fraction of strain-specific genes. The high-density tiling oligonucleotide array-based CGH allowed us to identify the complete array of DNA sequences unique to an invasive compared to a commensal GBS isolate. A denser and shorter oligonucleotide array design followed by a verification oligonucleotide array can be used for identifying even single nucleotide polymorphisms in the genome [35]. However, CGH-based genome comparisons depend on the availability of sequenced genomes, and moreover, strain-specific genes identified are confined to the sequenced genome. We were not able to detect and identify potential virulence genes that are likely to exist in other unsequenced pathogenic GBS strains. Given the pan-genome nature of the GBS species where the pool of variable genes are extremely large [20], future genome comparisons of any two strains are likely to rely on a cheap and fast direct sequencing approach such as pyrosequencing [52]. This new approach will eliminate the limitations of using $\mathrm{CGH}$.

Once a set of candidate genes are identified by a genomic comparison, an even more critical step is to evaluate the role that these genes play in disease pathogenesis. This can be done by large-scale association studies, bioinformatic prediction, or biological functional analyses. Bioinformatics prediction requires databases with solid structural and functional information on biological molecules. Functional approaches are often limited to the presence of a characterizable virulence phenotype. Comparing gene frequencies among bacterial isolates collected from different sources, for example, disease-causing and commensal isolates, using statistical association, can provide insight into the relative importance of a gene sequence in pathogenesis and transmission. The number of isolates and diversity of the collections are important in determining the significance of observations made and in ensuring that there is sufficient power to detect associations. Large population-based samples are required to minimize the identification of spurious associations that often arise with small sample comparisons. Including commensal isolates (i.e., nondisease-causing strains) for study is an integral part of this approach to understand bacterial pathogenesis. The LOS microarray platform is a robust system, adaptable to a wide variety of bacterial pathogens, for detecting the presence or absence of a candidate gene in thousands of isolates efficiently, thus providing a truly high throughput system to evaluate genes in the postgenome era.

\section{ACKNOWLEDGMENTS}

This research was supported by NIH Grant R01AI51675 (BF). We thank Dr. Carol J. Baker of Baylor College of Medicine and Terrence A. Kurzynski of Wisconsin State Laboratory of Hygiene for providing some of the GBS strains used in this study. We would like also to thank Maneesh Dave and Elizabeth Marie Levin for help with some of the LOS screening.

\section{REFERENCES}

[1] M. M. Farley, "Group B streptococcal disease in nonpregnant adults," Clinical Infectious Diseases, vol. 33, no. 4, pp. 556-561, 2001.

[2] A. Schuchat, "Epidemiology of group B streptococcal disease in the United States: shifting paradigms," Clinical Microbiology Reviews, vol. 11, no. 3, pp. 497-513, 1998.

[3] A. Schuchat, "Group B streptococcus," Lancet, vol. 353, no. 9146, pp. 51-56, 1999. 
[4] H. Dele Davies, C. Adair, A. McGeer, et al., "Antibodies to capsular polysaccharides of group B streptococcus in pregnant Canadian women: relationship to colonization status and infection in the neonate," Journal of Infectious Diseases, vol. 184, no. 3, pp. 285-291, 2001.

[5] N. Jones, K. A. Oliver, J. Barry, et al., "Enhanced invasiveness of bovine-derived neonatal sequence type 17 group B streptococcus is independent of capsular serotype," Clinical Infectious Diseases, vol. 42, no. 7, pp. 915-924, 2006.

[6] L. H. Harrison, J. A. Elliott, D. M. Dwyer, et al., "Serotype distribution of invasive group B streptococcal isolates in Maryland: implications for vaccine formulation. Maryland Emerging Infections Program," Journal of Infectious Diseases, vol. 177, no. 4, pp. 998-1002, 1998.

[7] C. S. Lachenauer, D. L. Kasper, et al., "Serotypes VI and VIII predominate among group B streptococci isolated from pregnant Japanese women," Journal of Infectious Diseases, vol. 179, no. 4, pp. 1030-1033, 1999.

[8] D. F. Zaleznik, M. A. Rench, S. Hillier, et al., "Invasive disease due to group B streptococcus in pregnant women and neonates from diverse population groups," Clinical Infectious Diseases, vol. 30, no. 2, pp. 276-281, 2000.

[9] P. Bidet, N. Brahimi, C. Chalas, Y. Aujard, and E. Bingen, "Molecular characterization of serotype III Group Bstreptococcus isolates causing neonatal meningitis," Journal of Infectious Diseases, vol. 188, no. 8, pp. 1132-1137, 2003.

[10] J. F. Bohnsack, S. Takahashi, S. R. Detrick, et al., "Phylogenetic classification of serotype III group B streptococci on the basis of hylB gene analysis and DNA sequences specific to restriction digest pattern type III-3," Journal of Infectious Diseases, vol. 183, no. 11, pp. 1694-1697, 2001.

[11] M. Hauge, C. Jespersgaard, K. Poulsen, and M. Kilian, "Population structure of streptococcus agalactiae reveals an association between specific evolutionary lineages and putative virulence factors but not disease," Infection and Immunity, vol. 64, no. 3, pp. 919-925, 1996.

[12] J. M. Musseer, S. J. Mattingly, R. Quentin, A. Goudeau, and R. K. Selander, "Identification of a high-virulence clone of type III streptococcus agalactiae (group B streptococcus) causing invasive neonatal disease," Proceedings of the National Academy of Sciences of the United States of America, vol. 86, no. 12, pp. 4731-4735, 1989.

[13] S. Takahashi, E. E. Adderson, Y. Nagano, N. Nagano, M. R. Briesacher, and J. F. Bohnsack, "Identification of a highly encapsulated, genetically related group of invasive type III group B streptococci," Journal of Infectious Diseases, vol. 177, no. 4, pp. 1116-1119, 1998.

[14] R. Helmig, N. Uldbjerg, J. Boris, and M. Kilian, "Clonal analysis of streptococcus agalactiae isolated from infants with neonatal sepsis or meningitis and their mothers and from healthy pregnant women," Journal of Infectious Diseases, vol. 168, no. 4, pp. 904-909, 1993.

[15] E. E. Adderson, S. Takahashi, Y. Wang, J. Armstrong, D. V. Miller, and J. F. Bohnsack, "Subtractive hybridization identifies a novel predicted protein mediating epithelial cell invasion by virulent serotype III group B streptococcus agalactiae," Infection and Immunity, vol. 71, no. 12, pp. 6857-6863, 2003.

[16] M. A. Herbert, C. J. E. Beveridge, and N. J. Saunders, "Bacterial virulence factors in neonatal sepsis: group B streptococous," Current Opinion in Infectious Diseases, vol. 17, no. 3, pp. 225229, 2004.

[17] G. Lindahl, M. Stålhammar-Carlemalm, and T. Areschoug, "Surface proteins of streptococcus agalactiae and related pro- teins in other bacterial pathogens," Clinical Microbiology Reviews, vol. 18, no. 1, pp. 102-127, 2005.

[18] V. Nizet, "Streptococcal $\beta$-hemolysins: genetics and role in disease pathogenesis," Trends in Microbiology, vol. 10, no. 12, pp. 575-580, 2002.

[19] V. Nizet and C. E. Rubens, "Pathogenic mechanisms and virulence factors of group B Streptococci," in Gram-Positive Pathogens, V. A. Fischetti, R. P. Novick, J. J. Ferretti, D. A. Portnoy, and J. I. Rood, Eds., ASM Press, Washington, DC, USA, 2000.

[20] H. Tettelin, V. Masignani, M. J. Cieslewicz, et al., "Genome analysis of multiple pathogenic isolates of streptococcus agalactiae: implications for the microbial "pan-genome"', Proceedings of the National Academy of Sciences of the United States of America, vol. 102, no. 39, pp. 13950-13955, 2005.

[21] P. Glaser, C. Rusniok, C. Buchrieser, et al., "Genome sequence of streptococcus agalactiae, a pathogen causing invasive neonatal disease," Molecular Microbiology, vol. 45, no. 6, pp. 14991513, 2002.

[22] M. A. Herbert, C. J. E. Beveridge, D. McCormick, et al., "Genetic islands of streptococcus agalactiae strains NEM316 and 2603VR and their presence in other Group B Streptococcal strains," BMC Microbiology, vol. 5, p. 31, 2005.

[23] H. Tettelin, V. Masignani, M. J. Cieslewicz, et al., "Complete genome sequence and comparative genomic analysis of an emerging human pathogen, serotype V streptococcus agalactiae," Proceedings of the National Academy of Sciences of the United States of America, vol. 99, no. 19, pp. 12391-12396, 2002.

[24] L. Zhang, B. Foxman, S. D. Manning, P. Tallman, and C. F. Marrs, "Molecular epidemiologic approaches to urinary tract infection gene discovery in uropathogenic Escherichia coli," Infection and Immunity, vol. 68, no. 4, pp. 2009-2015, 2000.

[25] G. K. Schoolnik, "Functional and comparative genomics of pathogenic bacteria," Current Opinion in Microbiology, vol. 5, no. 1, pp. 20-26, 2002.

[26] T. S. Whittam and A. C. Bumbaugh, "Inferences from wholegenome sequences of bacterial pathogens," Current Opinion in Genetics and Development, vol. 12, no. 6, pp. 719-725, 2002.

[27] S. J. Bliss, S. D. Manning, P. Tallman, et al., "Group B streptococcus colonization in male and nonpregnant female university students: a cross-sectional prevalence study," Clinical Infectious Diseases, vol. 34, no. 2, pp. 184-190, 2002.

[28] B. Foxman, B. Gillespie, S. D. Manning, et al., "Incidence and duration of group B streptococcus by serotype among male and female college students living in a single dormitory," American Journal of Epidemiology, vol. 163, no. 6, pp. 544-551, 2006.

[29] S. D. Manning, M. D. Pearlman, P. Tallman, C. L. Pierson, and B. Foxman, "Frequency of antibiotic resistance among group B streptococcus isolated from healthy college students," Clinical Infectious Diseases, vol. 33, no. 12, pp. e137-e139, 2001.

[30] S. D. Manning, P. Tallman, C. J. Baker, B. Gillespie, C. F. Marrs, and B. Foxman, "Determinants of co-colonization with group B streptococcus among heterosexual college couples," Epidemiology, vol. 13, no. 5, pp. 533-539, 2002.

[31] S. D. Manning, B. Foxman, C. L. Pierson, P. Tallman, C. J. Baker, and M. D. Pearlman, "Correlates of antibiotic-resistant group B streptococcus isolated from pregnant women," Obstetrics and Gynecology, vol. 101, no. 1, pp. 74-79, 2003.

[32] S. M. Borchardt, J. H. DeBusscher, P. A. Tallman, et al., "Frequency of antimicrobal resistance among invasive and colonizing Group B Streptococcal isolates," BMC Infectious Diseases, vol. 6, p. $57,2006$. 
[33] R. C. Lancefield, M. McCarty, and W. N. Everly, "Multiple mouse protective antibodies directed against group B streptococci. Special reference to antibodies effective against protein antigens," Journal of Experimental Medicine, vol. 142, no. 1, pp. 165-179, 1975.

[34] S. M. Borchardt, B. Foxman, D. O. Chaffin, et al., "Comparison of DNA dot blot hybridization and lancefield capillary precipitin methods for group B streptococcal capsular typing," Journal of Clinical Microbiology, vol. 42, no. 1, pp. 146-150, 2004.

[35] T. J. Albert, D. Dailidiene, G. Dailide, et al., "Mutation discovery in bacterial genomes: metronidazole resistance in $\mathrm{He}$ licobacter pylori," Nature Methods, vol. 2, no. 12, pp. 951-953, 2005.

[36] J. Yang, J. Wang, Z.-J. Yao, Q. Jin, Y. Shen, and R. Chen, "GenomeComp: a visualization tool for microbial genome comparison," Journal of Microbiological Methods, vol. 54, no. 3, pp. 423-426, 2003.

[37] S. F. Altschul, T. L. Madden, A. A. Schaffer, et al., "Gapped BLAST and PSI-BLAST: a new generation of protein database search programs," Nucleic Acids Research, vol. 25, no. 17, pp. 3389-3402, 1997.

[38] L. Zhang, U. Srinivasan, C. F. Marrs, D. Ghosh, J. R. Gilsdorf, and B. Foxman, "Library on a slide for bacterial comparative genomics," BMC Microbiology, vol. 4, p. 12, 2004.

[39] L. Zhang, B. Foxman, J. R. Gilsdorf, and C. F. Marrs, "Bacterial genomic DNA isolation for microarray analysis using sonication," BioTechniques, vol. 39, no. 5, pp. 640-644, 2005.

[40] L. Zhang, B. W. Gillespie, C. F. Marrs, and B. Foxman, "Optimization of a fluorescent-based phosphor imaging dot blot DNA hybridization assay to assess $E$. coli virulence gene profiles," Journal of Microbiological Methods, vol. 44, no. 3, pp. 225-233, 2001.

[41] R. L. Tatusov, M. Y. Galperin, D. A. Natale, and E. V. Koonin, "The COG database: a tool for genome-scale analysis of protein functions and evolution," Nucleic Acids Research, vol. 28, no. 1, pp. 33-36, 2000.

[42] A. L. Jones, K. M. Knoll, and C. E. Rubens, "Identification of streptococcus agalactiae virulence genes in the neonatal rat sepsis model using signature-tagged mutagenesis," Molecular Microbiology, vol. 37, no. 6, pp. 1444-1455, 2000.

[43] H. Gutekunst, B. J. Eikmanns, and D. J. Reinscheid, "The novel fibrinogen-binding protein $\mathrm{FbsB}$ promotes streptococcus agalactiae invasion into epithelial cells," Infection and Immunity, vol. 72, no. 6, pp. 3495-3504, 2004.

[44] I.-M. Jonsson, G. Pietrocola, P. Speziale, M. Verdrengh, and A. Tarkowski, "Role of fibrinogen-binding adhesin expression in septic arthritis and septicemia caused by streptococcus agalactiae," Journal of Infectious Diseases, vol. 192, no. 8, pp. 14561464, 2005.

[45] I. Benz and M. A. Schmidt, "Never say never again: protein glycosylation in pathogenic bacteria," Molecular Microbiology, vol. 45, no. 2, pp. 267-276, 2002.

[46] S. K. Ghosh, J. Zhao, M. C. Philogene, A. Alzaharani, S. Rane, and A. Banerjee, "Pathogenic consequences of Neisseria gonorrhoeae pilin glycan variation," Microbes and Infection, vol. 6, no. 7, pp. 693-701, 2004.

[47] C. M. Szymanski and B. W. Wren, "Protein glycosylation in bacterial mucosal pathogens," Nature Reviews Microbiology, vol. 3, no. 3, pp. 225-237, 2005.

[48] M. Matsuoka and T. Sasaki, "Inactivation of macrolides by producers and pathogens," Current Drug Targets - Infectious Disorders, vol. 4, no. 3, pp. 217-240, 2004.
[49] L. M. Quirós, R. J. Carbajo, and J. A. Salas, "Inversion of the anomeric configuration of the transferred sugar during inactivation of the macrolide antibiotic oleandomycin catalyzed by a macrolide glycosyltransferase," FEBS Letters, vol. 476, no. 3, pp. 186-189, 2000.

[50] M. M. Pettigrew, B. Foxman, C. F. Marrs, and J. R. Gilsdorf, "Identification of the lipooligosaccharide biosynthesis gene lic $2 B$ as a putative virulence factor in strains of nontypeable Haemophilus influenzae that cause otitis media," Infection and Immunity, vol. 70, no. 7, pp. 3551-3556, 2002.

[51] A. T. Maurelli, R. E. Fernández, C. A. Bloch, C. K. Rode, and A. Fasano, "“Black holes" and bacterial pathogenicity: a large genomic deletion that enhances the virulence of Shigella spp. and enteroinvasive Escherichia coli," Proceedings of the National Academy of Sciences of the United States of America, vol. 95, no. 7, pp. 3943-3948, 1998.

[52] M. Ronaghi, "Pyrosequencing sheds light on DNA sequencing," Genome Research, vol. 11, no. 1, pp. 3-11, 2001. 


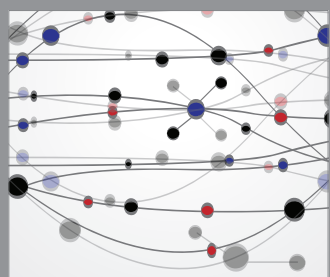

The Scientific World Journal
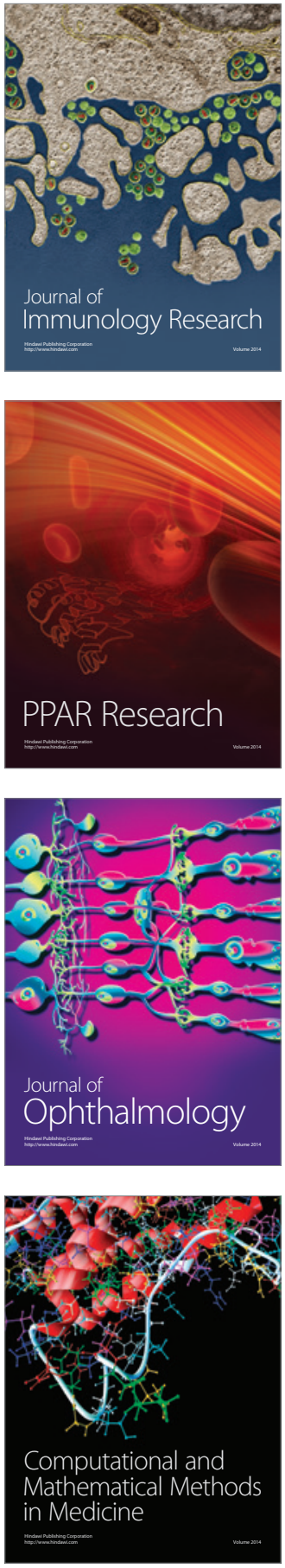

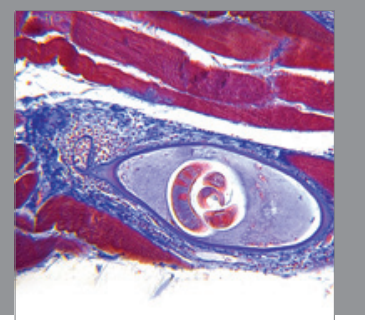

Gastroenterology

Research and Practice
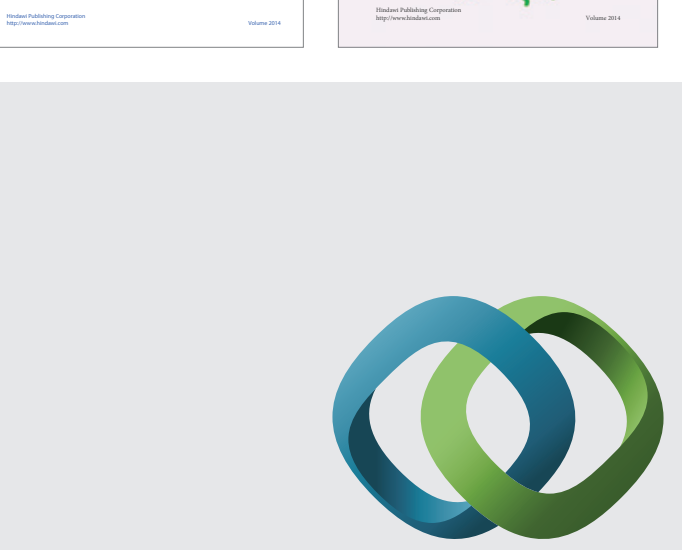

\section{Hindawi}

Submit your manuscripts at

http://www.hindawi.com
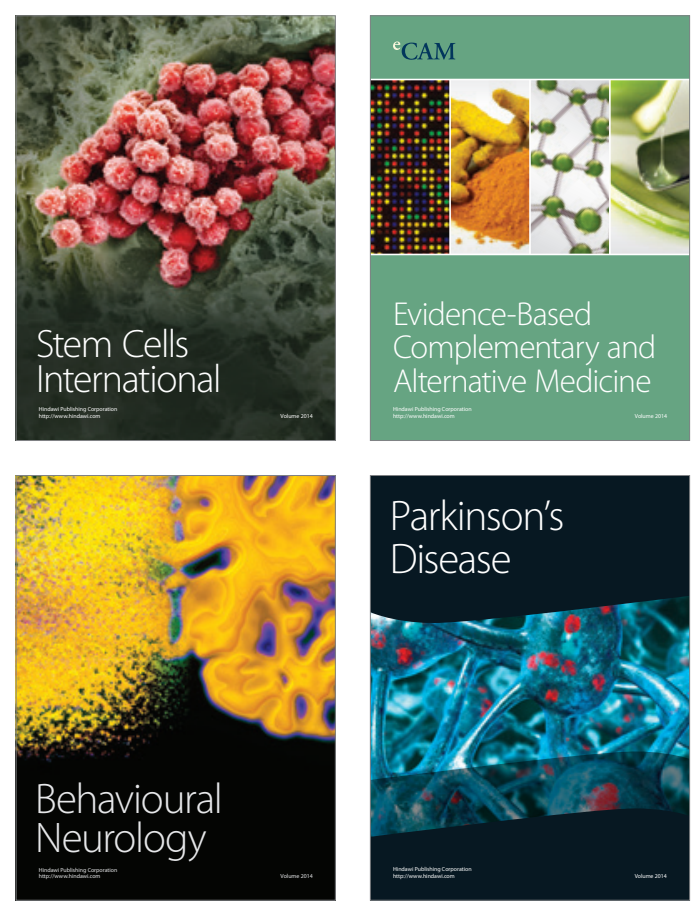

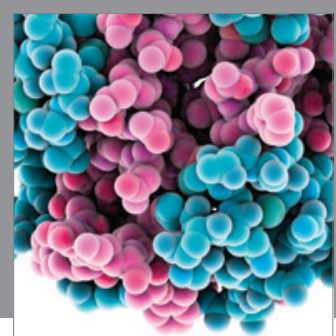

Journal of
Diabetes Research

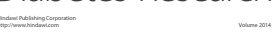

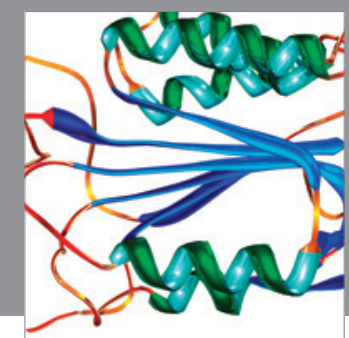

Disease Markers
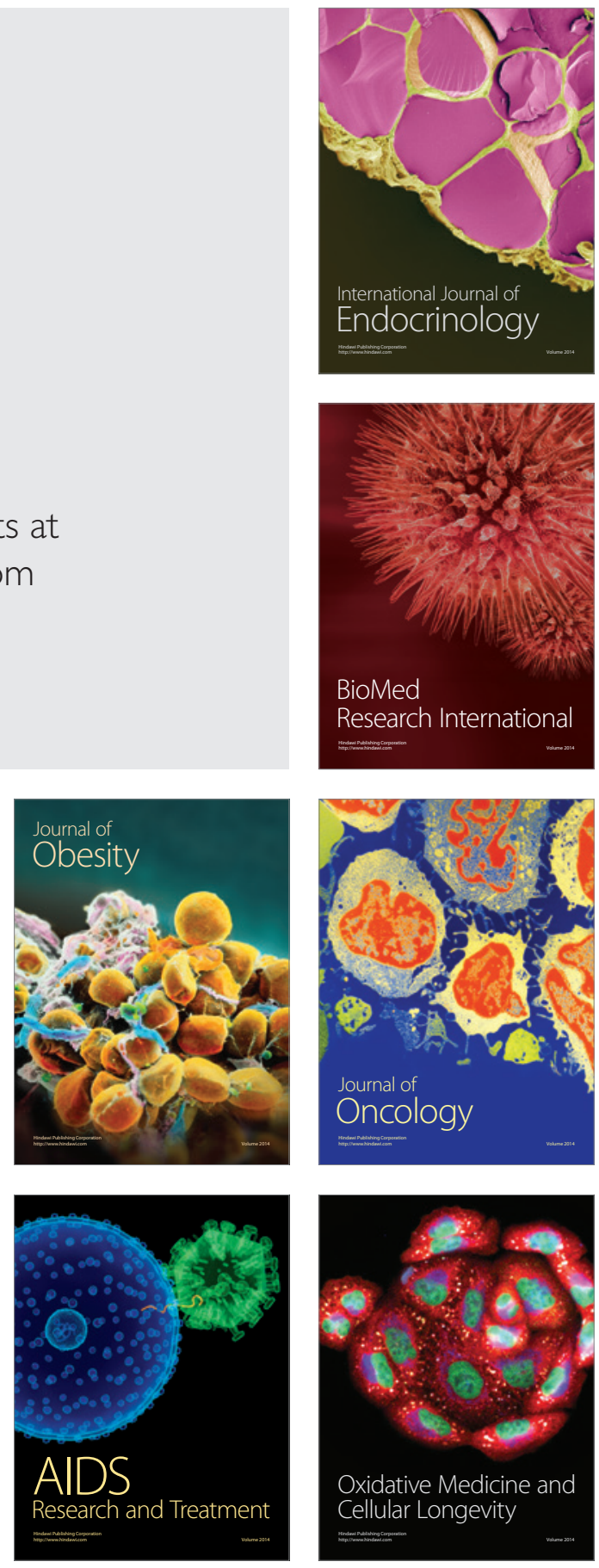